\title{
Precision Measurement of Lithium Flux in Cosmic Rays with the Alpha Magnetic Spectrometer on the International Space Station
}

\author{
Laurent Derome* on behalf of the AMS collaboration \\ Laboratoire de Physique Subatomique et de Cosmologie (LPSC), CNRS/IN2P3 and Université \\ Grenoble-Alpes, F-38026 Grenoble, France \\ E-mail: laurent.deromedlpsc.in2p3.fr
}

Lithium nuclei in cosmic rays are produced by the spallation of heavier cosmic rays on the interstellar medium. Thus, the abundance of lithium constitutes a very sensitive observable for the modeling of cosmic rays propagation in the Galaxy. A precision measurement of the lithium flux with rigidities from $2 \mathrm{GV}$ to $3 \mathrm{TV}$ by AMS, is presented.

The 34th International Cosmic Ray Conference,

30 July- 6 August, 2015

The Hague, The Netherlands

${ }^{*}$ Speaker. 


\section{Introduction}

Lithium is, as Boron and Beryllium, produced by spallation processes by heavier nuclei during their propagation in our Galaxy. As secondary component or even tertiary (since it can also be produced by the spallation of secondaries) the abundance of lithium is sensitive to the propagation history of cosmic rays and them can be used to constrain the parameters describing the diffusion, convection or reacceleration of cosmic rays in the Galaxy [1]. Previous measurements of lithium flux as been reported from balloon and space experiments [2].

In this presentation we report the precise measurement of the lithium flux in primary cosmic rays in the rigidity range from $2 \mathrm{GV}$ to $3 \mathrm{TV}$ based on data collected by the Alpha Magnetic Spectrometer (AMS) during the first 30 months (May 19, 2011 to November 26, 2013) of operation onboard the International Space Station (ISS).

\section{Detector}

AMS is a general purpose high energy spectrometer in space. The layout and description of the detector are presented in Ref. [3]. The key elements used in this measurement are the permanent magnet, the silicon tracker, four planes of time of flight (TOF) scintillation counters, and the array of anticoincidence counters (ACC). AMS also contains a transition radiation detector (TRD), a ring imaging Čerenkov detector (RICH), and an electromagnetic calorimeter (ECAL). The AMS coordinate system is concentric with the magnet and above, below, and downward-going refer to the AMS coordinate system. Timing, location, and orientation are provided by GPS units affixed to AMS and to the ISS. The detector performance has been steady over time.

The tracker [4] has nine layers, the first (L1) at the top of the detector, the second (L2) just above the magnet, six (L3 to L8) within the bore of the magnet, and the last (L9) just above the ECAL. L2 to L8 constitute the inner tracker. The tracker accurately determines the trajectory of cosmic rays by multiple measurements of the coordinates. Together, the tracker and the magnet measure the rigidity $R=p / Z$ of charged cosmic rays.

Each layer of the tracker also provides an independent measurement of the absolute value of the charge $|Z|$ of the cosmic ray.

Two planes of TOF counters [5] are located above L2 and two planes are located below the magnet. The velocity measurement is used to discriminate between upward- and downward-going particles. The pulse heights of the two upper layers are combined to provide another independent measurement of the absolute charge as are the pulse heights from the two lower planes.

Monte Carlo simulated events were produced using a dedicated program developed by the collaboration based on the GEANT-4.10.1 package [6]. The program simulates electromagnetic and hadronic interactions of particles in the material of AMS and generates detector responses. In particular, helium-nuclear interactions were modeled with the DPMJET-II.5 package [7] above and INCL++ package [8] below incident energy of $5 \mathrm{GeV} /$ nucleon. The digitization of the signals is simulated precisely according to the measured characteristics of the electronics. The simulated events then undergo the same reconstruction as used for the data. The Monte Carlo event samples have sufficient statistics such that they do not contribute to the errors. 


\section{Selection}

In the first 30 months $\left(7.96 \times 10^{7} \mathrm{~s}\right)$ AMS collected $4.1 \times 10^{10}$ cosmic ray events. The collection time used in this analysis includes only those seconds during which the detector was in normal operating conditions, the AMS was pointing within $40^{\circ}$ of the local zenith, the trigger live time exceeded 50\%, and the ISS was outside of the South Atlantic Anomaly. Due to the influence of the geomagnetic field, this collection time for primary cosmic rays increases with increasing rigidity becoming constant at $6.29 \times 10^{7} \mathrm{~s}$ above $30 \mathrm{GV}$ [11].

The main identification power is provided by the selection of events with charge compatible with $Z=3$ in the inner tracker, as it is shown in Fig. 1. To improve the rejection and to remove the events which interacted within the detector, the charges measured by L1, the upper TOF, the inner tracker, the lower TOF, and L9 are also required to be compatible with $Z=3$.

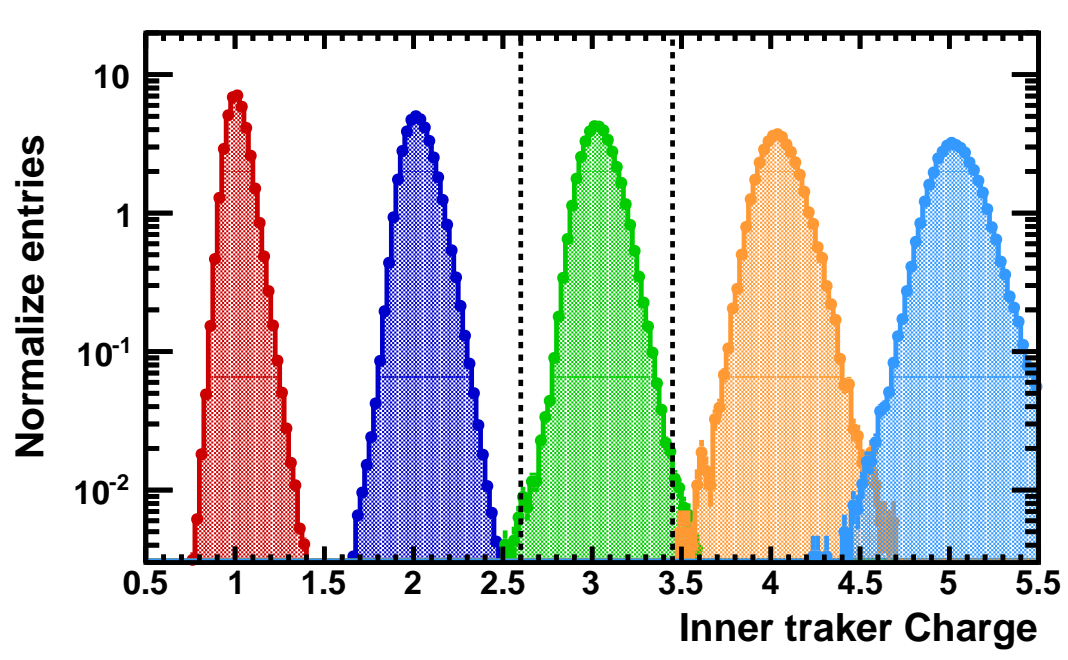

Figure 1: Charge distributions obtained from data in the inner tracker for nuclei from $Z=1$ to $Z=5$. The dashed lines indicate the values used to select the lithium sample in the inner tracker.

Due to the multiple independent measurements of the absolute charge, the selected sample contains only a small contamination of particles which had $Z \neq 3$. The contamination from $Z<3$ particles is completely negligible after the tracker and the TOF selection. Small contamination is observed from higher charge cosmic rays $(Z>3)$ which interact and produce lithium on the top of AMS. Fig. 2 shows the distribution of charge measured in L1 for events selected as $Z=3$ in the inner tracker and in the TOF system. The charge selection in L1 is used to remove the contamination arising from interactions between tracker L1 and L2. Comparison of the L1 charge distribution between data and MC simulation is used to estimate the small contamination from interaction above L1.

In order to optimize the measurement, two tracker pattern selections are used in this analysis: first selected sample with events having a track passing through L1 and L9 in order to have the optimized resolution at the highest rigidities, and a second sample with events passing trough only L1 and the inner. The later pattern, corresponding to a larger detector acceptance, is used to optimize the event statistics. Further selections are made by requiring the track to satisfy additional track 


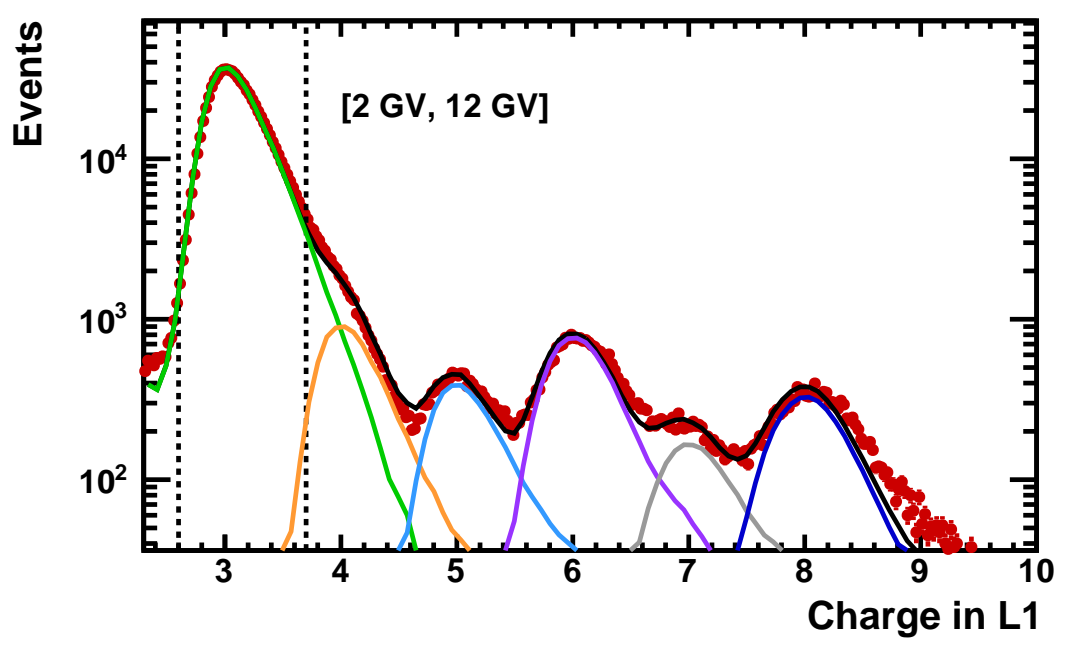

Figure 2: Charge distribution (red dots) measured in tracker $\mathrm{L} 1$ for the $Z=3$ sample obtained after charge selection in the inner tracker and the TOF system and with rigidity ranging from 2 to $12 \mathrm{GV}$. The distribution is fitted with templates (lines) corresponding to the charge distributions for nuclei from $Z=3$ (green), $Z=4$ (orange), $Z=5$ (light blue), $Z=6$ (violet), $Z=7$ (gray) and $Z=8$ (dark blue). The overall fit is represented by the black line. The selection region used to remove the contamination from interaction between tracker L1 and L2 is indicated with the vertical dashed lines.

fitting quality criteria such as a $\chi^{2} /$ d.f. $<20$ in the bending coordinate. To select only primary cosmic rays, the measured rigidity is required to be greater than a factor of 1.2 times the maximum geomagnetic cutoff within the AMS field of view. The cutoff was calculated by backtracing [9] particles from the top of AMS out to 50 Earth's radii using the most recent IGRF [10] geomagnetic model.

Most importantly, several independent analyses were performed on the same data sample by different study groups. The current results of those analyses are consistent with the ones presented here.

\section{Analysis}

The lithium flux $\Phi_{i}$ for the $i^{\text {th }}$ rigidity bin $\left(R_{i}, R_{i}+\Delta R_{i}\right)$ is

$$
\Phi_{i}=\frac{N_{i}}{A_{i} \varepsilon_{i} T_{i} \Delta R_{i}}
$$

where $N_{i}$ is the number of events corrected with the rigidity resolution function in the bin $\left(R_{i}, R_{i}+\right.$ $\left.\Delta R_{i}\right), A_{i}$ is the effective acceptance for an isotropic flux, $\varepsilon_{i}$ is the trigger efficiency, and $T_{i}$ is the collection time. The lithium flux was measured in 68 bins, $i=1$ to 68 , from $1.9 \mathrm{GV}$ to $3 \mathrm{TV}$ with bin widths chosen according to the rigidity resolution. The effective acceptance $A_{i}$ was calculated using the Monte Carlo simulation and then corrected for the small differences on event selection efficiencies found between the data and the Monte Carlo.

The trigger efficiency $\varepsilon_{i}$ was measured to be above $99.5 \%$ in the whole range of rigidity. The Monte Carlo simulation agrees with the measured trigger efficiency within $0.5 \%$. 
The bin-to-bin migration of events was corrected using the two unfolding procedures described in Ref. [11].

Extensive studies were made of the systematic errors. These errors include the uncertainties in the trigger efficiency, the acceptance, the background contamination, the lithium isotopic composition, the geomagnetic cutoff factor, the event selection, the unfolding, the rigidity resolution function, and the absolute rigidity scale.

Most importantly, several independent analyses were performed on the same data sample by different study groups. The results of those analyses are consistent with this Letter.

\section{Results}

The AMS measured lithium flux $\Phi$ including statistical errors and systematic errors are presented. The contribution of individual sources to the systematic error are added in quadrature to arrive at the total systematic uncertainty.

\section{References}

[1] I. A. Grenier, J. H. Black and A. W. Strong, Annu. Rev. Astron. Astrophys, 53, 199-246 (2015); P. Blasi, Braz. J. Phys. 44, 426 (2014); P. Blasi, Astron. Astrophys. Rev. 21, 70 (2013); Andrew W. Strong, Igor V. Moskalenko, and Vladimir S. Ptuskin, Annual Review of Nuclear and Particle Science 57, 285-327 (2007).

[2] G. A. De Nolfo et al., Advances in Space Research 38, 1558 (2006); E. Juliusson, The Astrophysical Journal 191, 331 (1974); C. D. Orth et al., The Astrophysical Journal 226, 1147 (1978); Data from D. Maurin, F. Melot, R. Taillet, A\&A 569, A32 (2014)

[3] A. Kounine, Int. J. Mod. Phys. E 211230005 (2012); S. Rosier-Lees, in Proceedings of Astroparticle Physics TEVPA/IDM, Amsterdam, 2014 (to be published); S. Ting, Nucl. Phys. B, Proc. Suppl. 243-244, 12 (2013); B. Bertucci, Proc. Sci., EPS-HEP, (2011) 67; M. Incagli, AIP Conf. Proc. 1223, 43 (2010); R. Battiston, Nucl. Instrum. Methods Phys. Res., Sect. A 588, 227 (2008).

[4] B. Alpat et al., Nucl. Instrum. Methods Phys. Res., Sect. A 613, 207 (2010).

[5] V. Bindi et al., Nucl. Instrum. Methods Phys. Res., Sect. A 743, 22 (2014) and references therein.

[6] J. Allison et al., IEEE Trans. Nucl. Sci. 53, 270 (2006); S. Agostinelli et al., Nucl. Instrum. Methods Phys. Res., Sect. A 506, 250 (2003).

[7] J. Ranft, Phys. Rev. D 51, 64 (1995).

[8] A. Boudard, J. Cugnon, J.-C. David, S. Leray, and D. Mancusi Phys. Rev. C 87014606 (2013); S. Leray, D. Mancusi, P. Kaitaniemi, J. C. David, A. Boudard, B. Braunn, and J. Cugnon J. Phys.: Conf. Series 420, 012065 (2013).

[9] J. Alcaraz et al., Phys. Lett. B 484, 10 (2000).

[10] C. C. Finlay et al., Geophys. J. Int. 183, 1216 (2010). We have used data from IGRF-12 (2015), currently available at http://www.ngdc.noaa.gov/IAGA/vmod/igrf.html (unpublished).

[11] M. Aguilar et al., Phys. Rev. Lett. 114, 171103(2015) . 